Bangl. J. Vet. Med. (2008). 6 (1): 07-12

\title{
ISOLATION, IDENTIFICATION AND CHARACTERIZATION OF SALMONELLA FROM DUCK
}

\author{
T. Mondal ${ }^{1}$, M. S. R. Khan ${ }^{1 *}$, M. Alam², M. Purakayastha ${ }^{1}$, M. Das ${ }^{1}$ and M. P. Siddique ${ }^{1}$ \\ ${ }^{1}$ Department of Microbiology and Hygiene, Faculty of Veterinary Science, Bangladesh Agricultural University, \\ Mymensingh 2202, Bangladesh, ${ }^{2}$ Enteric Microbiology Laboratory, Laboratory Sciences Division, ICDDR,B, \\ Dhaka, Bangladesh, * Corresponding author’s e-mail: msrkhan001@yahoo.com
}

\begin{abstract}
The present study was performed with the aim to isolate and identify Salmonella organism from diarrhoeic and apparently healthy ducks and to characterize duck Salmonella by biochemical test. Antibiotic sensitivity analysis of duck Salmonella was performed. A total of 65 cloacal samples were collected from ducks of three different regions such as Char Nilokkhiya, BAU Poultry Farm and Boyra, Mymensingh. Out of 65 samples 9 (13.07\%) were found positive. The antibiotic sensitivity pattern showed that the duck isolates were highly sensitive to ciprofloxacin, kanamycin, nalidixic acid, co-trimoxazole and cephalexin but these isolates were highly resistant to chloramphenicol.
\end{abstract}

Key words: Salmonella, duck, isolation, identification, characterization

\section{INTRODUCTION}

Salmonellae are Gram negative, short plump shaped rods, nonsporeforming, noncapsulated, aerobic and facultatively anaerobic organisms and classified under the family Enterobacteriaceae (OIE Manual, 2006). In 1919, Rettger reported a disease which was observed in ducklings from which he isolated an organism typical of the Salmonella group. The following year Rettger and Scoville (1920) described the organism in detail and named it Bacterium anatis. However, Price and Berry (1962) have shown that Salmonella anatum is the most important infectious agent in case of duck but number of other Salmonellae has been isolated from cases of the disease. In case of duck, the disease is extremely acute and fatal. The affected individuals appear weak and sluggish and remain close to the heating pipes. Few of the ducklings die after they are from three to four weeks of age; the highest mortality occurring within the first week or 7-10 days. Examination of the dead ducklings revealed no lesions of any pathogenic condition except, paleness of the tissues as a whole and light body weight (Merchant and Packer, 1967). Salmonella infections are also important as both a cause of clinical disease in duck and as a source of food-borne transmission of disease to humans. Heavy economic losses occur due to morbidity, mortality, reduced egg and meat production in duck. Mortality may vary from $10 \%$ to $80 \%$ or higher in severe outbreaks (Kumar and Kaushi, 1988; Kaura et al., 1990; Kleven and Yoder, 1998). The transmission of Salmonella from duck meat and egg is a great concern from the public health point of view. For the prevention and control of any microbial disease, prior isolation, identification and characterization of that particular etiological agent in a country is a pre-requisite. We carried out this study to isolate, identify and characterize Salmonella from duck using various cultural, morphological, biochemical, and antibiotic sensitivity test.

\section{MATERIALS AND METHODS}

The present research was conducted between November 2006 to October 2007 in the Bacteriology laboratory of the Department of Microbiology and Hygiene, Faculty of Veterinary Science, Bangladesh Agricultural University (BAU), Mymensingh.

\section{Collection of sample}

A total of 65 cloacal swab samples, 24 from Bangladesh Agricultural University (BAU) Poultry Farm, 22 from Boyra, Mymensingh and 19 from Char Nilokkhiya were used in this study. The samples were collected from 40 apparently healthy ducks and 25 diarrhoeic ducks for bacteriological study. 
The ducks were the breeds of Khaki Campbell and Indian Runner and age ranged from 2 to 3 months without considering any sex. Sterile cotton swab and sterile Bacto-selenite broth in sterile glass tubes were carried to the sampling areas and samples were collected from the mucosa of the cloaca of the ducks. The swabs collected aseptically were transferred immediately into Bacto selenite broth and test tubes containing swab samples were then immediately brought to the bacteriology laboratory, Department of Microbiology and Hygiene, BAU and subjected to cultural study.

\section{Cultivation and isolation of Salmonella}

Each of the collected cloacal swabs was inoculated into freshly prepared selenite broth. Then the tubes were marked properly and incubated at $37^{\circ} \mathrm{C}$ for 24 hours aerobically in bacteriological incubator. The incubated tubes were then examined for growth of bacteria. Smears were prepared from each of the test tubes and the smears were fixed. The fixed smears were stained with Gram's Method of staining and examined under microscope at 100 magnifications using immersion oil. In presence of gram negative rods in the smears, the materials from the tube corresponding to the smears were streaked into MacConkey agar, Salmonella-Shigella agar and Brilliant green agar separately. The plates were then incubated at $37^{\circ} \mathrm{C}$ for 24 hours and the plates containing characteristic colonies of Salmonella are selected. Motility test and Gram's staining test are performed to identify the plates containing Salmonella accurately. Subculturing in Salmonella-Shigella agar was performed from the suspected plates containing Salmonella to obtain a pure culture. These pure isolates obtained in this way were used for further study (Cheesbrough, 1985).

\section{Morphological characterization by Gram's staining method}

A small colony from the representative Salmonella colonies was picked up from SS, MC and BGA plates with a bacteriological loop, smeared on separate glass slide and fixed by gentle heating. Crystal violet was then applied on each smear to stain for two minutes and then washed with running water. Few drops of Gram's iodine was then added to act as mordent for one minute and then again washed with running tap water. Acetone alcohol was then added (acts as decolorizer) for few seconds. After washing with water, safranin was added as counter stain and allowed to stain for 2 minutes. Then the slides were washed with water, blotted and dried in air and then examined under microscope with high power objective (100X) using immersion oil (Merchant and Packer, 1967).

\section{Motility test using hanging drop slide}

The motility test was performed to differentiate motile bacteria from non-motile one. Before performing the test, a pure culture of the organism was allowed to grow in Nutrient Broth (NB). One drop of cultured broth was placed on the clean cover-slip and was placed invertedly over the concave depression of the hanging drop slide to make hanging drop preparation. Vaseline was used around the concave depression of the hanging drop slide for better attachment of the cover-slip and to prevent evaporation of the fluid by air current. The hanging drop slide was then examined carefully under high power objective (100X) of a compound light microscope using immersion oil. The motile and non-motile organisms were identified by observing motility in contrasting with swinging movement of bacteria (Merchant and Packer, 1967). The motile bacteria with swinging movement were identified as Salmonella.

\section{Biochemical characterization}

Isolated organisms with supporting growth characteristics of Salmonella on various media were maintained on SS and BGA and were subjected to the following biochemical tests named sugar fermentation test, MR-VP reaction and indole reaction.

\section{Sugar fermentation test}

The carbohydrate fermentation test was performed by inoculating a loopful of thick bacterial culture into the tubes containing five basic sugars (dextrose, maltose, sucrose, lactose, and mannitol) and incubated at $37^{\circ} \mathrm{C}$ for 24 hours. Acid production was indicated by the change of media from pink to yellow color and gas production was indicated by the appearance of gas bubbles in the inverted Durham's fermentation tubes (Cheesbrough, 1985). 


\section{Characterization of Salmonella}

\section{Methyl red test}

The test was conducted by inoculating a colony of the test organism in $0.5 \mathrm{ml}$ sterile glucose phosphate peptone broth. After overnight incubation at $37^{\circ} \mathrm{C}$, a drop of methyl red solution was added. A red coloration is positive and indicates an acid $\mathrm{p}^{\mathrm{H}}$ of 4.5 or less resulting from the fermentation of glucose. A yellow coloration is negative (Cheesbrough, 1985).

\section{Voges-Proskauer test}

An amount of $2 \mathrm{ml}$ of sterile glucose phosphate peptone water were inoculated with the $5 \mathrm{ml}$ of test organisms. It was incubated at $37^{\circ} \mathrm{C}$ aerobically for 48 hours. A very small amount (knifepoint) of creatine was added and mixed. $3 \mathrm{ml}$ of sodium hydroxide were added and shaked well. The bottle cap was removed and left for an hour at room temperature. It was observed closely for the slow development of pink color for positive cases. In negative cases, there was no development of pink color (Cheesbrough, 1985).

\section{Indole test}

Two $\mathrm{ml}$ of peptone water was inoculated with $5 \mathrm{ml}$ of bacterial culture and incubated for 48 hours. Kovac's reagent $(0.5 \mathrm{ml})$ was added and mixed thoroughly. The tube was then allowed to stand for a while. The appearance of red color on the whole medium was considered as a positive test for the production of indole by the organisms (Cheesbrough, 1985).

\section{Antibiogram study of the isolated Salmonella spp.}

Susceptibility of the isolated Salmonellae to different antibacterial agents was performed through disc diffusion method to determine the drug sensitivity pattern. The antibacterial discs used were erythromycin, amoxicillin, cephalexin, chloramphenicol, co-trimoxazole, kanamycin, ciprofloxacin and nalidixic acid. Salmonella isolates were grown overnight on BGA and the overnight cultured isolates were inoculated into NB and poured on BGA and spreaded uniformly with the help of sterile glass spreader. Antibacterial discs were applied aseptically to the surface of the plate at an appropriate arrangement with the help of sterile forceps and incubated at $37^{\circ} \mathrm{C}$ for 24 hours, aerobically (Carter, 1979).

\section{RESULTS AND DISCUSSION}

During this study out of 65 samples 9 were found to be positive to Salmonella and among the positive samples, 4 were from BAU Poultry Farm, 3 were from Boyra, Mymensingh and 2 were from Char Nilokkhiya. The percentages of positive samples from those areas were $16.6 \%, 13.63 \%$ and $10.52 \%$, respectively (Table 1 ).

Table 1. Isolation of Salmonella from cloacal swab samples collected from apparently healthy and diarrhoeic ducks of BAU Poultry Farm, Boyra, Mymensingh and Char Nilokkhiya

\begin{tabular}{|c|c|c|c|c|c|c|c|}
\hline \multirow[t]{3}{*}{ Name of the place } & \multirow{3}{*}{$\begin{array}{l}\text { No. of } \\
\text { sample } \\
\text { tested }\end{array}$} & \multicolumn{2}{|c|}{ Cultural examination } & \multicolumn{2}{|c|}{ Biochemical examination } & \multicolumn{2}{|c|}{$\begin{array}{l}\text { Total positive } \\
\text { Salmonella }\end{array}$} \\
\hline & & Positive & Negative & Positive & Negative & & \\
\hline & & & & & & No. & $\%$ \\
\hline BAU Poultry Farm & 24 & 4 & 20 & 4 & 20 & 4 & 16.66 \\
\hline Boyra, Mymensingh & 22 & 3 & 19 & 3 & 19 & 3 & 13.63 \\
\hline Char Nilokkhiya & 19 & 2 & 17 & 2 & 17 & 2 & 10.52 \\
\hline Total & 65 & 9 & 56 & 9 & 56 & 9 & 13.07 \\
\hline
\end{tabular}

After cultural examination we found that the positive samples showed the characteristic colonies in different media such as SS, MC, BGA (Table 2, Plate 1, Plate 2 and Plate 3). Morphological characterization revealed that the isolates were Gram negative, short rod shaped organisms and after motility test we also found that they were motile (Table 2 and Plate 4). 
T. Mondal and others

Table 2. Results of cultural, staining and morphological characteristics of the isolated Salmonella from duck

\begin{tabular}{|llllll|}
\hline \multirow{2}{*}{$\begin{array}{l}\text { Source of } \\
\text { isolates }\end{array}$} & Colony characteristics & \multicolumn{2}{c|}{ Staining characters } & Motility \\
\cline { 2 - 4 } & SS agar & MC agar & BGA & \\
\hline D1Fa & Opaque, translucent, & Pale, colorless smooth, & Pale pink color & Gram negative, \\
& colorless, smooth, & transparent, raised & colonies against & short rod shaped & \\
& round colonies & colonies & a pinkish & & \\
& & & background & & + \\
D2Fb & Do & Do & Do & Do & + \\
D3Fc & Do & Do & Do & Do & + \\
D4Ca & Do & Do & Do & Do & + \\
D5Cb & Do & Do & Do & Do & + \\
D6Bb & Do & Do & Do & Do & + \\
D7Bc & Do & Do & Do & Do & + \\
D8Bd & Do & Do & Do & Do & + \\
D9Be & Do & Do & Do & & + \\
\end{tabular}

D1Fa, D2Fb and D3Fc = Cloacal swab sample of duck from BAU Poultry Farm; SS = Salmonella-Shigella agar; D4Ca and $\mathrm{D} 5 \mathrm{Cb}=$ Cloacal swab samples of duck from Char Nilokkhya; MC = MacConkey agar; D6Bb, D7Bc, D8Bd and D9Be = Cloacal swab samples of duck from Boyra, Mymensingh; BGA = Briliant Green agar; + = Positive

After biochemical examination we observed that all of the isolates fermented dextrose, maltose and mannitol with the production of acid and gas but did not ferment lactose and sucrose (Plate 5). Those were negative to Voges-Proskauer test and Indole test with the exception of D1Fa and D6Bb but the isolates were positive to Methyl Red test (Plate 6). From the antibiogram study, it was revealed that the isolates were highly sensitive to ciprofloxacin, kanamycin, nalidixic acid, and cephalexin while moderately sensitive to kanamycin, nalidixic acid, co-trimoxazole, cephalexin, erythromycin and amoxicillin. The isolates were less sensitive to erythromycin, amoxicillin and chloramphenicol whereas they were resistant to chloramphenicol (Fig. 1).

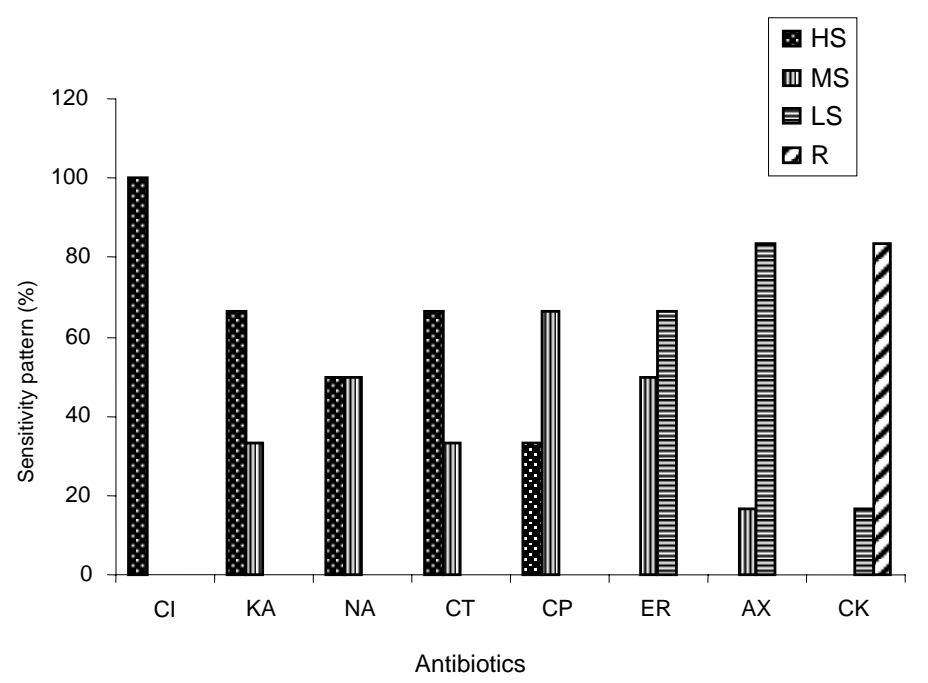

Fig. 1. Bar diagrammatic pattern of antibiotic sensitivity and resistance pattern of duck Salmonella isolates.

$\mathrm{CI}=$ Ciprofloxacin, $\mathrm{CT}=$ Co-trimoxazole, $\mathrm{AX}=$ Amoxicillin, $\mathrm{KA}=$ Kanamycin, $\mathrm{CP}=$ Cephalexin, $\mathrm{CK}=$ Chloramphenicol, $\mathrm{NA}=$ Nalidixic acid, ER = Erythromycin, HS = Highly sensitive, $\mathrm{MS}=$ Moderately sensitive, $\mathrm{LS}=$ Less sensitive and $\mathrm{R}=$ Resistant. 


\section{Characterization of Salmonella}

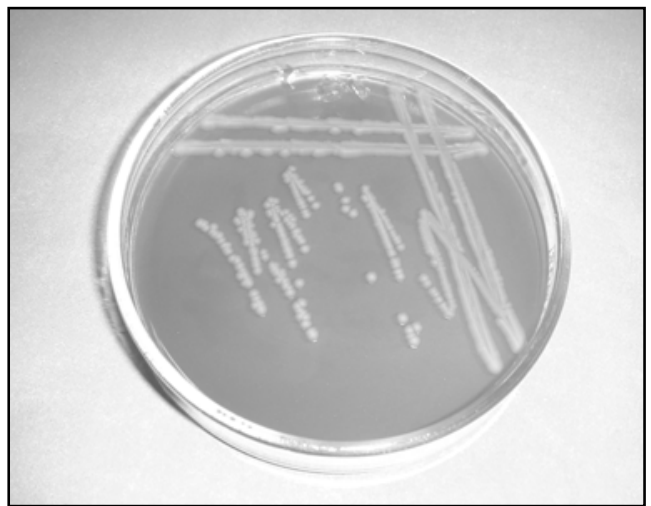

Plate 1. Growth of Salmonella on MacConkey agar.

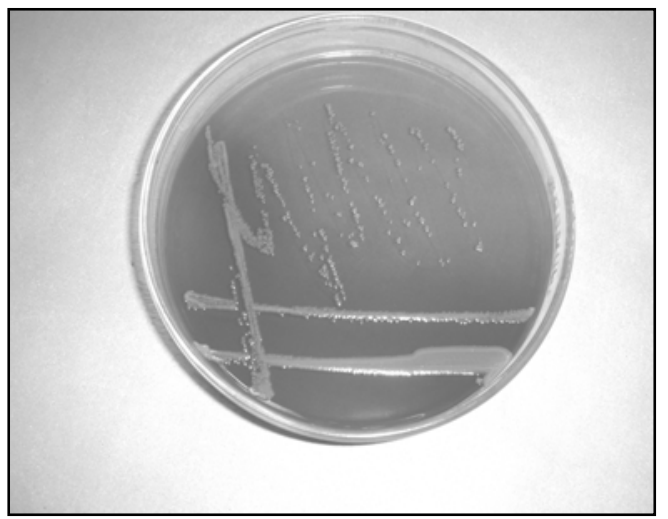

Plate 3. Growth of Salmonella on Brilliant green agar.

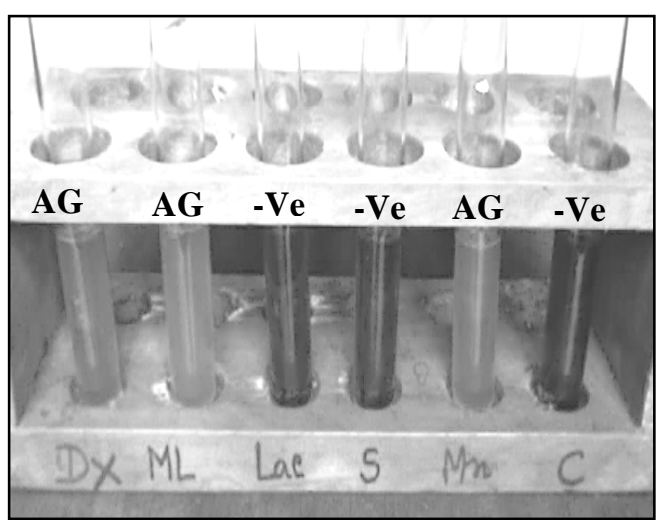

Plate 5. Results of sugar fermentation test of Salmonella.

$(\mathrm{DX}=$ Dextrose; $\mathrm{ML}=$ Maltose; Lac $=$ Lactose; $\mathrm{S}=$ Sucrose; $\mathrm{Mn}=$ Mannitol; $\mathrm{C}=$ Control; $\mathrm{AG}=$ Acid and Gas; $-\mathrm{Ve}=$ Negative)

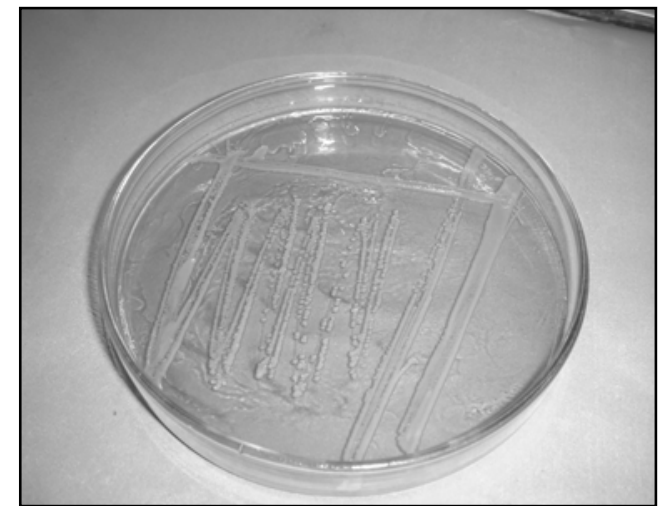

Plate 2. Growth of Salmonella on SalmonellaShigella agar.

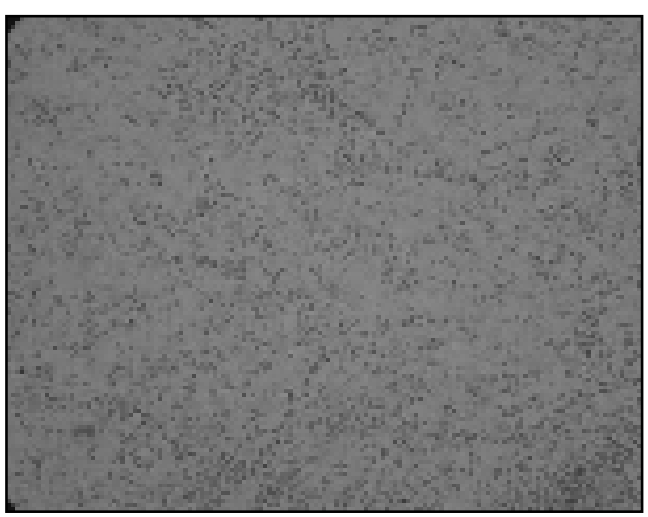

Plate 4. Salmonella spp. showing Gram negative small rods arranged singly or pairs (Gram's staining).

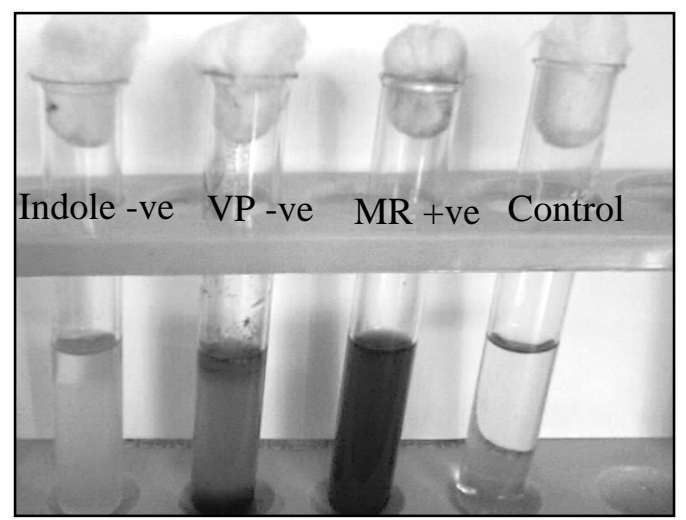

Plate 6. Results of Indole and Methyl Red-Voges Proskauer test. 


\section{REFERENCES}

1. Carter GR (1979). Diagnostic Procedures in Veterinary Bacteriology and Mycology. $3^{\text {rd }}$ edn., Charles E Thomas Publisher, USA. pp. 398-417.

2. Cheesbrough M (1985). Medical Laboratory Manual for Tropical Countries. Vol. 2. Microbiology. pp. $400-480$.

3. Kleven SH and Yoder HW (1998). Mycoplasmosis. In: HG Purchase, LH Arp, CH Domermuth and JE Pearson (Eds). A Laboratory Manual for the Isolation and Identification of Avian Pathogens. $4^{\text {th }}$ edn., American Association of Avian Pathologists, Kenett Square. pp. 74-88.

4. Kumar A and Kaushi RK (1988). Investigation of fowl typhoid in Haryana state. Indian Journal of Poultry Science 23: 104-106.

5. Merchant IA and Packer RA (1967). Veterinary Bacteriology and Virology. $7^{\text {th }}$ edn., The Iowa University Press, Ames, Iowa, USA. pp. 286-306.

6. OIE (2006). Salmonellosis. Office International des Epizooties. http://www.oie.int/chapter X.4. T

7. $\quad$ Price JI and Berry LJ (1962). Salmonella infection in white pekin duck. Avian Diseases 6: 145-147.

8. Rettger LF and Scoville MM (1920). Bacterium anatum. Journal of Infectious Diseases 26: 215-229. 